\title{
The estimates of diagonally dominant degree and eigenvalues distributions for the Schur complements of matrices
}

Yao-tang $\mathrm{Li}^{*}$ and Feng Wang

\section{"Correspondence:}

liyaotang@ynu.edu.cn

School of Mathematics and

Statistics, Yunnan University,

Kunming, Yunnan 650091, P.R. China

\begin{abstract}
By applying the properties of Schur complement and some inequality techniques, some new estimates of diagonally dominant degree on the Schur complement of matrices are obtained, which improve the main results of Liu (SIAM J. Matrix Anal. Appl. 27:665-674, 2005) and Liu (Linear Algebra Appl. 432:1090-1104, 2010). As an application, we present some new distribution theorems for eigenvalues of the Schur complement. Finally, we give a numerical example to illustrate the theory results.
\end{abstract}

MSC: $15 \mathrm{~A} 45 ; 15 \mathrm{~A} 48$

Keywords: matrix; Schur complement; diagonally dominant degree; eigenvalue distribution

\section{Introduction}

Let $\mathbb{C}^{n \times n}$ denote the set of all $n \times n$ complex matrices, $N=\{1,2, \ldots, n\}$ and $A=\left(a_{i j}\right) \in \mathbb{C}^{n \times n}$ $(n \geq 2)$. Denote

$$
\begin{aligned}
& R_{i}(A)=\sum_{j \neq i}\left|a_{i j}\right|, \quad C_{i}(A)=\sum_{j \neq i}\left|a_{j i}\right|, \quad i \in N, \\
& N_{r}(A)=\left\{i:\left|a_{i i}\right|>R_{i}(A), i \in N\right\}, \quad N_{c}(A)=\left\{i:\left|a_{i i}\right|>C_{i}(A), i \in N\right\} .
\end{aligned}
$$

We call $A$ a strictly diagonally dominant matrix (abbreviated $S D_{n}$ ) if

$$
\left|a_{i i}\right|>R_{i}(A), \quad \forall i \in N .
$$

$A$ is called an Ostrowski matrix (abbreviated $O S_{n}$ ) (see [1]) if

$$
\left|a_{i i}\right|\left|a_{j j}\right|>R_{i}(A) R_{j}(A), \quad \forall i, j \in N, i \neq j .
$$

As in [2], for all $i \in N$ and $\alpha \in[0,1]$, we call $\left|a_{i i}\right|-R_{i}(A),\left|a_{i i}\right|-\alpha R_{i}(A)-(1-\alpha) C_{i}(A)$ and $\left|a_{i i}\right|-\left[R_{i}(A)\right]^{\alpha}\left[C_{i}(A)\right]^{1-\alpha}$ the $i$ th dominant degree, $\alpha$-dominant degree and product $\alpha$-dominant degree of $A$, respectively.

For $\beta \subseteq N$, denote by $|\beta|$ the cardinality of $\beta$ and $\bar{\beta}=N / \beta$. If $\beta, \gamma \subseteq N$, then $A(\beta, \gamma)$ is the submatrix of $A$ lying in the rows indexed by $\beta$ and the columns indicated by $\gamma$. In

\section{Springer}

(c) $2013 \mathrm{Li}$ and Wang; licensee Springer. This is an Open Access article distributed under the terms of the Creative Commons Attribution License (http://creativecommons.org/licenses/by/2.0), which permits unrestricted use, distribution, and reproduction in any medium, provided the original work is properly cited. 
particular, $A(\beta, \beta)$ is abbreviated to $A(\beta)$. If $A(\beta)$ is nonsingular, then

$$
A / \beta=A / A(\beta)=A(\bar{\beta})-A(\bar{\beta}, \beta)[A(\beta)]^{-1} A(\beta, \bar{\beta})
$$

is called the Schur complement of $A$ with respect to $A(\beta)$.

The comparison matrix of $A, \mu(A)=\left(\alpha_{i j}\right)$ is defined by

$$
\alpha_{i j}= \begin{cases}\left|a_{i j}\right|, & \text { if } i=j, \\ -\left|a_{i j}\right|, & \text { if } i \neq j .\end{cases}
$$

A matrix $A$ is called an $M$-matrix if there exist a nonnegative matrix $B$ and a real number $s>\rho(B)$ such that $A=s I-B$, where $\rho(B)$ is the spectral radius of $B$. It is well known that $A$ is an $H$-matrix if and only if $\mu(A)$ is an $M$-matrix, and if $A$ is an $M$-matrix, then the Schur complement of $A$ is also an $M$-matrix and $\operatorname{det} A>0$ (see [3]). We denote by $H_{n}$ and $M_{n}$ the sets of $H$-matrices and $M$-matrices, respectively.

The Schur complement has been proved to be a useful tool in many fields such as control theory, statistics and computational mathematics, and many works have been done on it (see [4-8]). Meanwhile, studying the locations of eigenvalues of the Schur complement of matrices is of great significance as shown in [2, 3, 9-14]. In this paper, we present some new estimates of diagonally dominant degree on the Schur complement of matrices and use them to study the distributions for the eigenvalues of the Schur complement of matrices.

The paper is organized as follows. In Section 2, we give several new estimates of the diagonally dominant degree, the $\alpha$-diagonally dominant degree and product $\alpha$-diagonally dominant degree on the Schur complement of matrices. In Section 3, several new distribution theorems for eigenvalues of the Schur complements are obtained. In Section 4, we present a numerical example to illustrate the theory results.

\section{Estimates of diagonally dominant degree for the Schur complement}

In this section, we present several new estimates for the diagonally dominant degree, the $\alpha$-diagonally dominant degree and product $\alpha$-diagonally dominant degree of the Schur complement of matrices.

Lemma 1 [5] If $A \in H_{n}$, then $[\mu(A)]^{-1} \geq\left|A^{-1}\right|$.

Lemma 2 [5] If $A$ is an $S D_{n}$ or $A$ is an $O S_{n}$, then $A \in H_{n}$, i.e., $\mu(A) \in M_{n}$.

Lemma 3 [3] If $A$ is an $S D_{n}$ or $A$ is an $O S_{n}$ and $\beta \subseteq N$, then the Schur complement of $A$ is an $S D_{|\bar{\beta}|}$ or an $O S_{|\bar{\beta}|}$, where $\bar{\beta}=N-\beta$ is the complement of $\beta$ in $N$, and $|\bar{\beta}|$ is the cardinality of $\bar{\beta}$.

Lemma 4 [2] Let $a>b, c>b, b>0$ and $0 \leq \alpha \leq 1$. Then

$$
a^{\alpha} c^{1-\alpha} \geq(a-b)^{\alpha}(c-b)^{1-\alpha}+b .
$$


Theorem 1 Let $A=\left(a_{i j}\right) \in \mathbb{C}^{n \times n}, \beta=\left\{i_{1}, i_{2}, \ldots, i_{k}\right\} \subseteq N_{r}(A) \neq \phi, \bar{\beta}=\left\{j_{1}, j_{2}, \ldots, j_{l}\right\}, k<n$, and let $A / \beta=\left(a_{t s}^{\prime}\right)$. Then for all $1 \leq t \leq l$,

$$
\left|a_{t t}^{\prime}\right|-R_{t}(A / \beta) \geq\left|a_{j t j t}\right|-R_{j t}(A)+\delta_{j t} \geq\left|a_{j t j t}\right|-R_{j t}(A),
$$

and

$$
\left|a_{t t}^{\prime}\right|+R_{t}(A / \beta) \leq\left|a_{j j_{t}}\right|+R_{j_{t}}(A)-\delta_{j_{t}} \leq\left|a_{j j_{t} t}\right|+R_{j_{t}}(A)
$$

where

$$
\begin{aligned}
& \delta_{j_{t}}=\min _{1 \leq u \leq k} \frac{\left|a_{i_{u} i_{u}}\right|-P_{i_{u}}(A)}{\left|a_{i_{u} i_{u}}\right|} \sum_{v=1}^{k}\left|a_{j_{t} i_{v}}\right|, \quad r=\max _{1 \leq u \leq k} \frac{\sum_{v=1}^{l}\left|a_{i_{u} j_{v}}\right|}{\left|a_{i_{u} i_{u}}\right|-\sum_{v \neq u}^{k}\left|a_{i_{u} i_{v}}\right|}, \\
& P_{i_{u}}(A)=r \sum_{v \neq u}^{k}\left|a_{i_{u} i_{v}}\right|+\sum_{v=1}^{l}\left|a_{i_{u} j_{v}}\right|, \quad i_{u} \in \beta, 1 \leq u \leq k .
\end{aligned}
$$

Proof Since $\beta \subseteq N_{r}(A) \neq \phi$, then $A(\beta) \in H_{k}, \mu(A(\beta)) \in M_{k}$. From Lemma 1 and Lemma 2, we have

$$
[\mu(A(\beta))]^{-1} \geq\left|[A(\beta)]^{-1}\right| .
$$

Thus, for $1 \leq t \leq l$,

$$
\begin{aligned}
& \left|a_{t t}^{\prime}\right|-R_{t}(A / \beta) \\
& =\left|a_{t t}^{\prime}\right|-\sum_{s \neq t}^{l}\left|a_{t s}^{\prime}\right| \\
& \geq\left|a_{j_{t} j_{t}}\right|-\sum_{s \neq t}^{l}\left|a_{j_{t} j_{s}}\right|-\sum_{s=1}^{l}\left(\left|a_{j_{t} i_{1}}\right|, \ldots,\left|a_{j_{t} i_{k}}\right|\right)[\mu(A(\beta))]^{-1}\left(\begin{array}{c}
\left|a_{i_{1} j_{s}}\right| \\
\vdots \\
\left|a_{i_{k} j_{s}}\right|
\end{array}\right) \\
& =\left|a_{j_{t} j_{t}}\right|-R_{j_{t}}(A)+\sum_{v=1}^{k}\left|a_{j_{t} t_{v}}\right|+\delta_{j_{t}}-\delta_{j_{t}}-\sum_{s=1}^{l}\left(\left|a_{j_{t} i_{1}}\right|, \ldots,\left|a_{j_{t} i_{k}}\right|\right)[\mu(A(\beta))]^{-1}\left(\begin{array}{c}
\left|a_{i_{1} j_{s}}\right| \\
\vdots \\
\left|a_{i_{k} j_{s}}\right|
\end{array}\right) \\
& =\left|a_{j_{t j t}}\right|-R_{j_{t}}(A)+\delta_{j t} \\
& +\frac{1}{\operatorname{det}[\mu(A(\beta))]} \operatorname{det}\left(\begin{array}{cccc}
\sum_{v=1}^{k}\left|a_{j t i_{v}}\right|-\delta_{j t} & -\left|a_{j t}\right| i_{1} \mid & \ldots & -\left|a_{j t i_{k}}\right| \\
-\sum_{s=1}^{l}\left|a_{i_{1} j_{s}}\right| & & & \\
\vdots & & \mu(A(\beta)) & \\
-\sum_{s=1}^{l}\left|a_{i_{k} j_{s}}\right| & &
\end{array}\right) \\
& \stackrel{\text { def. }}{=}\left|a_{j t j_{t}}\right|-R_{j_{t}}(A)+\delta_{j_{t}}+\frac{1}{\operatorname{det}[\mu(A(\beta))]} \operatorname{det} B \text {. }
\end{aligned}
$$

Similarly to the proof of Lemma 4 in [13], we can prove that $\operatorname{det} B \geq 0$. Thus, we obtain Inequation (1). Similarly, we can prove Inequation (2). 
Remark 1 Note that

$$
\frac{P_{i_{u}}(A)}{\left|a_{i_{u} i_{u}}\right|} \leq r \leq \frac{R_{i_{u}}(A)}{\left|a_{i_{u} i_{u}}\right|}, \quad 1 \leq u \leq k .
$$

This shows that Theorem 1 improves Theorem 1 of [13].

Theorem 2 Let $A=\left(a_{i j}\right) \in \mathbb{C}^{n \times n}, \beta=\left\{i_{1}, i_{2}, \ldots, i_{k}\right\} \subseteq N_{r}(A) \cap N_{c}(A) \neq \phi, \bar{\beta}=\left\{j_{1}, j_{2}, \ldots, j_{l}\right\}$, $k<n$, and let $A / \beta=\left(a_{t s}^{\prime}\right)$. Then for all $1 \leq t \leq l, 0 \leq \alpha \leq 1$,

$$
\left|a_{t t}^{\prime}\right|-\left(R_{t}(A / \beta)\right)^{\alpha}\left(C_{t}(A / \beta)\right)^{1-\alpha} \geq\left|a_{j t t_{t}}\right|-\left(R_{j t}(A)-\delta_{t}\right)^{\alpha}\left(C_{j_{t}}(A)-\delta_{t}^{T}\right)^{1-\alpha},
$$

and

$$
\left|a_{t t}^{\prime}\right|+\left(R_{t}(A / \beta)\right)^{\alpha}\left(C_{t}(A / \beta)\right)^{1-\alpha} \leq\left|a_{j t j_{t}}\right|+\left(R_{j t}(A)-\delta_{t}\right)^{\alpha}\left(C_{j_{t}}(A)-\delta_{t}^{T}\right)^{1-\alpha}
$$

where

$$
\begin{aligned}
& \delta_{t}=\min _{1 \leq \omega \leq k} \frac{\left|a_{i_{\omega} i_{\omega}}\right|-P_{i_{\omega}}(A)}{\left|a_{i_{\omega} i_{\omega}}\right|} \sum_{\nu=1}^{k}\left|a_{j t i_{v}}\right|, \quad \eta=\max _{1 \leq \omega \leq k} \frac{\sum_{v=1}^{l}\left|a_{i_{\omega} j_{\nu}}\right|}{\left|a_{i_{\omega} i_{\omega}}\right|-\sum_{\nu \neq \omega}^{k}\left|a_{i_{\omega} i_{v}}\right|}, \\
& \delta_{t}^{T}=\min _{1 \leq \omega \leq k} \frac{\left|a_{i_{\omega} i_{\omega}}\right|-Q_{i_{\omega}}(A)}{\left|a_{i_{\omega} i_{\omega}}\right|} \sum_{v=1}^{k}\left|a_{i_{v} j t}\right|, \quad \xi=\max _{1 \leq \omega \leq k} \frac{\sum_{v=1}^{l}\left|a_{j i_{\omega}}\right|}{\left|a_{i_{\omega} i_{\omega}}\right|-\sum_{v \neq \omega}^{k}\left|a_{i_{v} i_{\omega}}\right|}, \\
& P_{i_{\omega}}(A)=\eta \sum_{\nu \neq \omega}^{k}\left|a_{i_{\omega} i_{\nu}}\right|+\sum_{\nu=1}^{l}\left|a_{i_{\omega} j_{v}}\right|, \quad Q_{i_{\omega}}(A)=\xi \sum_{\nu \neq \omega}^{k}\left|a_{i_{\nu} i_{\omega}}\right|+\sum_{\nu=1}^{l}\left|a_{j \nu} i_{\omega}\right| .
\end{aligned}
$$

Proof Since $\beta \subseteq N_{r}(A) \neq \phi$, then $A(\beta) \in H_{k}, \mu(A(\beta)) \in M_{k}$. From Lemma 1 and Lemma 2, we have

$$
[\mu(A(\beta))]^{-1} \geq\left|[A(\beta)]^{-1}\right|
$$

Thus, for all $1 \leq t \leq l, 0 \leq \alpha \leq 1$,

$$
\begin{aligned}
\left|a_{t t}^{\prime}\right| & -\left(R_{t}(A / \beta)\right)^{\alpha}\left(C_{t}(A / \beta)\right)^{1-\alpha} \\
\geq & \left|a_{j_{t j} t}\right|-\left(\left|a_{j_{t} i_{1}}\right|, \ldots,\left|a_{j_{t} i_{k}}\right|\right)[\mu(A(\beta))]^{-1}\left(\begin{array}{c}
\left|a_{i_{1} j_{t}}\right| \\
\vdots \\
\left|a_{i_{k} j_{t}}\right|
\end{array}\right) \\
& -\left(\sum_{s \neq t}^{l}\left[\left|a_{j_{t} j_{s}}\right|+\left(\left|a_{j_{t} i_{1}}\right|, \ldots,\left|a_{j_{t} i_{k}}\right|\right)[\mu(A(\beta))]^{-1}\left(\begin{array}{c}
\left|a_{i_{1} j_{s}}\right| \\
\vdots \\
\left|a_{i_{k} j_{s}}\right|
\end{array}\right)\right]\right)^{\alpha} \\
& \times\left(\sum_{s \neq t}^{l}\left[\left|a_{j_{s j} j_{t}}\right|+\left(\left|a_{j_{s} i_{1}}\right|, \ldots,\left|a_{j_{s} i_{k}}\right|\right)[\mu(A(\beta))]^{-1}\left(\begin{array}{c}
\left|a_{i_{1} j_{t}}\right| \\
\vdots \\
\left|a_{i_{k} j_{t}}\right|
\end{array}\right)\right]\right)^{1-\alpha} .
\end{aligned}
$$


Let

$$
\zeta=\left(\left|a_{j t} i_{1}\right|, \ldots,\left|a_{j t}\right|\right)[\mu(A(\beta))]^{-1}\left(\begin{array}{c}
\left|a_{i_{1} j_{t}}\right| \\
\vdots \\
\left|a_{i_{k} j_{t}}\right|
\end{array}\right) .
$$

By the proof of Theorem 1, we have

$$
\sum_{s \neq t}^{l}\left[\left|a_{j t_{j}}\right|+\left(\left|a_{j t_{1}}\right|, \ldots,\left|a_{j t i_{k}}\right|\right)[\mu(A(\beta))]^{-1}\left(\begin{array}{c}
\left|a_{i_{1} j_{s}}\right| \\
\vdots \\
\left|a_{i_{k} j_{s}}\right|
\end{array}\right)\right] \leq R_{j t}(A)-\delta_{t}-\zeta .
$$

Similarly,

$$
\sum_{s \neq t}^{l}\left[\left|a_{j s j t}\right|+\left(\left|a_{j s_{1}}\right|, \ldots,\left|a_{j s i_{k}}\right|\right)[\mu(A(\beta))]^{-1}\left(\begin{array}{c}
\left|a_{i_{1} j_{t}}\right| \\
\vdots \\
\left|a_{i_{k} j_{t} \mid}\right|
\end{array}\right)\right] \leq C_{j t}(A)-\delta_{t}^{T}-\zeta .
$$

By Lemma 4, we have

$$
\begin{aligned}
\left|a_{t t}^{\prime}\right| & -\left(R_{t}(A / \beta)\right)^{\alpha}\left(C_{t}(A / \beta)\right)^{1-\alpha} \\
& \geq\left|a_{j_{t j} j_{t}}\right|-\zeta-\left(R_{j_{t}}(A)-\delta_{t}-\zeta\right)^{\alpha}\left(C_{j_{t}}(A)-\delta_{t}^{T}-\zeta\right)^{1-\alpha} \\
& \geq\left|a_{j j_{j} t}\right|-\zeta-\left[\left(R_{j_{t}}(A)-\delta_{t}\right)^{\alpha}\left(C_{j_{t}}(A)-\delta_{t}^{T}\right)^{1-\alpha}-\zeta\right] \\
& =\left|a_{j t j_{t}}\right|-\left(R_{j t}(A)-\delta_{t}\right)^{\alpha}\left(C_{j_{t}}(A)-\delta_{t}^{T}\right)^{1-\alpha} .
\end{aligned}
$$

Thus, we obtain Inequation (3). Similarly, we can prove Inequation (4).

Remark 2 Note that

$$
\begin{aligned}
& \delta_{t}=\min _{1 \leq \omega \leq k} \frac{\left|a_{i_{\omega} i_{\omega}}\right|-P_{i_{\omega}}(A)}{\left|a_{i_{\omega} i_{\omega}}\right|} \sum_{\nu=1}^{k}\left|a_{j t i_{\nu}}\right| \geq \min _{1 \leq \omega \leq k} \frac{\left|a_{i_{\omega} i_{\omega}}\right|-R_{i_{\omega}}(A)}{\left|a_{i_{\omega} i_{\omega}}\right|} \sum_{v=1}^{k}\left|a_{j t i_{\nu}}\right|, \\
& \delta_{t}^{T}=\min _{1 \leq \omega \leq k} \frac{\left|a_{i_{\omega} i_{\omega}}\right|-Q_{i_{\omega}}(A)}{\left|a_{i_{\omega} i_{\omega}}\right|} \sum_{v=1}^{k}\left|a_{i_{\nu} j t}\right| \geq \min _{1 \leq \omega \leq k} \frac{\left|a_{i_{\omega} i_{\omega}}\right|-C_{i_{\omega}}(A)}{\left|a_{i_{\omega} i_{\omega}}\right|} \sum_{v=1}^{k}\left|a_{i_{v j}}\right| .
\end{aligned}
$$

This shows that Theorem 2 improves Theorem 2 of [2].

Similarly to the proof of Theorem 2, we can prove the following theorem.

Theorem 3 Let $A=\left(a_{i j}\right) \in \mathbb{C}^{n \times n}, \beta=\left\{i_{1}, i_{2}, \ldots, i_{k}\right\} \subseteq N_{r}(A) \cap N_{c}(A) \neq \phi, \bar{\beta}=\left\{j_{1}, j_{2}, \ldots, j_{l}\right\}$, $k<n$ and $A / \beta=\left(a_{t s}^{\prime}\right)$. Then for all $1 \leq t \leq l, 0 \leq \alpha \leq 1$,

$$
\begin{aligned}
& \left|a_{t t}^{\prime}\right|-\alpha R_{t}(A / \beta)-(1-\alpha) C_{t}(A / \beta) \\
& \quad \geq\left|a_{j j_{j} j_{t}}\right|-\alpha R_{j_{t}}(A)-(1-\alpha) C_{j_{t}}(A)+\alpha \delta_{t}+(1-\alpha) \delta_{t}^{T} \\
& \quad \geq\left|a_{j_{t j} j_{t}}\right|-\alpha R_{j_{t}}(A)-(1-\alpha) C_{j_{t}}(A),
\end{aligned}
$$


and

$$
\begin{aligned}
& \left|a_{t t}^{\prime}\right|+\alpha R_{t}(A / \beta)+(1-\alpha) C_{t}(A / \beta) \\
& \quad \leq\left|a_{j_{t j} j_{t}}\right|+\alpha R_{j_{t}}(A)+(1-\alpha) C_{j_{t}}(A)-\alpha \delta_{t}-(1-\alpha) \delta_{t}^{T} \\
& \quad \leq\left|a_{j_{t} j_{t}}\right|+\alpha R_{j_{t}}(A)+(1-\alpha) C_{j_{t}}(A) .
\end{aligned}
$$

\section{Distribution for eigenvalues of the Schur complement}

In this section, we present two new distribution theorems for eigenvalues of the Schur complement.

Lemma 5 [2] Let $A=\left(a_{i j}\right) \in \mathbb{C}^{n \times n}$ and $0 \leq \alpha \leq 1$. Then for any eigenvalue $\lambda$ of $A$, there exists $1 \leq t \leq n$ such that

$$
\left|\lambda-a_{t t}\right| \leq\left(R_{t}(A)\right)^{\alpha}\left(C_{t}(A)\right)^{1-\alpha}
$$

Theorem 4 Let $A=\left(a_{i j}\right) \in \mathbb{C}^{n \times n}, \beta=\left\{i_{1}, i_{2}, \ldots, i_{k}\right\} \subseteq N_{r}(A) \neq \phi, \bar{\beta}=\left\{j_{1}, j_{2}, \ldots, j_{l}\right\}$, and let $A / \beta=\left(a_{t s}^{\prime}\right)$. Then for any eigenvalue $\lambda$ of $A / \beta$, there exists $1 \leq t \leq l$ such that

$$
\left|\lambda-a_{j_{t} j_{t}}\right| \leq R_{j_{t}}(A)-\delta_{j_{t}} \leq R_{j_{t}}(A)
$$

Proof Let $\lambda$ be an eigenvalue of $A / \beta$. From the famous Gerschgorin circle theorem, we know that there exists $1 \leq t \leq l$ such that $\left|\lambda-a_{t t}^{\prime}\right| \leq R_{t}(A / \beta)$. Hence

$$
\begin{aligned}
0 \geq & \left|\lambda-a_{t t}^{\prime}\right|-R_{t}(A / \beta) \\
= & \left|\lambda-a_{j_{t j} j_{t}}+\left(a_{j_{t} i_{1}}, \ldots, a_{j_{t} i_{k}}\right)[A(\beta)]^{-1}\left(\begin{array}{c}
a_{i_{1} j_{t}} \\
\vdots \\
a_{i_{k} j_{t}}
\end{array}\right)\right| \\
& -\sum_{s=1, \neq t}^{l} \mid \begin{array}{c}
a_{j_{t} j_{s}}-\left(a_{j_{t} i_{1}}, \ldots, a_{j_{t} i_{k}}\right)[A(\beta)]^{-1}\left(\begin{array}{c}
a_{i_{1} j_{s}} \\
\vdots \\
a_{i_{k} j_{s}}
\end{array}\right) \mid \\
\geq
\end{array} \\
= & \left|\lambda-a_{j_{t} j_{t}}\right|-\sum_{s=1, \neq t}^{l}\left|a_{j_{t} j_{s}}\right|-\sum_{s=1}^{l}\left(\left|a_{j_{t} i_{1}}\right|, \ldots,\left|a_{j_{t} i_{k}}\right|\right)[\mu(A(\beta))]^{-1}\left(\begin{array}{c}
\left|a_{i_{1} j_{s}}\right| \\
\vdots \\
\left|a_{i_{k} j_{s}}\right|
\end{array}\right) \\
& -\sum_{s=1}^{l}\left(\left|a_{j_{t} i_{1}}\right|, \ldots,\left|a_{j_{t} i_{k}}\right|\right)[\mu(A(\beta))]^{-1}\left(\begin{array}{c}
\left|a_{i_{1} j_{s}}\right| \\
\vdots \\
\left|a_{i_{k} j_{s}}\right|
\end{array}\right) \\
& \left|a_{j_{t} j_{t}}\right|-R_{j_{t}}(A)+\delta_{j_{t}},
\end{aligned}
$$


that is

$$
\left|\lambda-a_{j t j_{t}}\right| \leq R_{j t}(A)-\delta_{j t} \leq R_{j t}(A)
$$

Thus, Inequation (5) holds.

Theorem 5 Let $A=\left(a_{i j}\right) \in \mathbb{C}^{n \times n}, \beta=\left\{i_{1}, i_{2}, \ldots, i_{k}\right\} \subseteq N_{r}(A) \cap N_{c}(A) \neq \phi, \bar{\beta}=\left\{j_{1}, j_{2}, \ldots, j_{l}\right\}$, $k<n$, and let $A / \beta=\left(a_{t s}^{\prime}\right)$. Then for any $0 \leq \alpha \leq 1$ and every eigenvalue $\lambda$ of $A / \beta$, there exists $1 \leq t \leq l$ such that

$$
\left|\lambda-a_{j_{t j} j_{t}}\right| \leq\left(R_{j_{t}}(A)-\delta_{t}\right)^{\alpha}\left(C_{j_{t}}(A)-\delta_{t}^{T}\right)^{1-\alpha} \leq\left(R_{j_{t}}(A)\right)^{\alpha}\left(C_{j_{t}}(A)\right)^{1-\alpha} .
$$

Proof Let $\lambda$ be an eigenvalue of $A / \beta$. By Lemma 5, we know that there exists $1 \leq t \leq l$ such that

$$
\left|\lambda-a_{t t}^{\prime}\right| \leq\left(R_{t}(A / \beta)\right)^{\alpha}\left(C_{t}(A / \beta)\right)^{1-\alpha}, \quad 0 \leq \alpha \leq 1 .
$$

Thus,

$$
\begin{aligned}
& 0 \geq\left|\lambda-a_{t t}^{\prime}\right|-\left(R_{t}(A / \beta)\right)^{\alpha}\left(C_{t}(A / \beta)\right)^{1-\alpha} \\
& =\left|\lambda-a_{j t_{t}}-\left(a_{j t_{1}}, \ldots, a_{j t_{k} i_{k}}\right)[A(\beta)]^{-1}\left(\begin{array}{c}
a_{i_{1} j_{t}} \\
\vdots \\
a_{i_{k} j_{t}}
\end{array}\right)\right| \\
& -\left[\sum_{s=1, \neq t}^{l}\left|a_{j t j_{s}}+\left(a_{j t i_{1}}, \ldots, a_{j t i_{k}}\right)[A(\beta)]^{-1}\left(\begin{array}{c}
a_{i_{1} j_{s}} \\
\vdots \\
a_{i_{k} j_{s}}
\end{array}\right)\right|\right]^{\alpha} \\
& \times\left[\sum_{s=1, \neq t}^{l}\left|a_{j_{s i t}}+\left(a_{j_{s i} i_{1}}, \ldots, a_{j_{s} i_{k}}\right)[A(\beta)]^{-1}\left(\begin{array}{c}
a_{i_{1} j_{t}} \\
\vdots \\
a_{i_{k} j_{t}}
\end{array}\right)\right|\right]^{1-\alpha} \\
& \geq\left|\lambda-a_{j_{t j} t}\right|-\left|\left(a_{j i_{1}}, \ldots, a_{j t_{k} i_{k}}\right)[A(\beta)]^{-1}\left(\begin{array}{c}
a_{i_{1} j_{t}} \\
\vdots \\
a_{i_{k} j_{t}}
\end{array}\right)\right| \\
& -\left(\sum_{s=1, \neq t}^{l}\left[\left|a_{j t j_{s}}\right|+\left|\left(a_{j t i_{1}}, \ldots, a_{j t i_{k}}\right)[A(\beta)]^{-1}\left(\begin{array}{c}
a_{i_{1} j_{s}} \\
\vdots \\
a_{i_{k} j_{s}}
\end{array}\right)\right|\right]\right)^{\alpha} \\
& \times\left(\sum_{s=1, \neq t}^{l}\left[\left|a_{j s j_{t}}\right|+\mid\left(a_{j_{s} i_{1}}, \ldots, a_{j_{s i} i_{k}}\right)[A(\beta)]^{-1}\left(\begin{array}{c}
a_{i_{1} j_{t}} \\
\vdots \\
a_{i_{k} j_{t}}
\end{array}\right)\right]\right)^{1-\alpha} .
\end{aligned}
$$


Similarly to the proof of Theorem 2, we can prove

$$
\begin{aligned}
& \left|\left(a_{j_{t} i_{1}}, \ldots, a_{j_{t} i_{k}}\right)[A(\beta)]^{-1}\left(\begin{array}{c}
a_{i_{1} j_{t}} \\
\vdots \\
a_{i_{k} j_{t}}
\end{array}\right)\right| \\
& +\left(\sum_{s=1, \neq t}^{l}\left[\left|a_{j_{t} j_{s}}\right|+\left|\left(a_{j_{t} i_{1}}, \ldots, a_{j_{t} i_{k}}\right)[A(\beta)]^{-1}\left(\begin{array}{c}
a_{i_{1} j_{s}} \\
\vdots \\
a_{i_{k} j_{s}}
\end{array}\right)\right|\right]\right)^{\alpha} \\
& \times\left(\sum_{s=1, \neq t}^{l}\left[\left|a_{j_{s} j_{t}}\right|+\left|\left(a_{j_{s} i_{1}}, \ldots, a_{j_{s} i_{k}}\right)[A(\beta)]^{-1}\left(\begin{array}{c}
a_{i_{1} j_{t}} \\
\vdots \\
a_{i_{k} j_{t}}
\end{array}\right)\right|\right]\right)^{1-\alpha} \\
& \leq\left(R_{j_{t}}(A)-\delta_{t}\right)^{\alpha}\left(C_{j_{t}}(A)-\delta_{t}^{T}\right)^{1-\alpha} \text {. }
\end{aligned}
$$

Therefore, we have

$$
0 \geq\left|\lambda-a_{t t}^{\prime}\right|-\left(R_{t}(A / \beta)\right)^{\alpha}\left(C_{t}(A / \beta)\right)^{1-\alpha} \geq\left|\lambda-a_{j_{t j} j_{t}}\right|-\left(R_{j_{t}}(A)-\delta_{t}\right)\left(C_{j_{t}}(A)-\delta_{t}^{T}\right)^{1-\alpha} .
$$

That is, Inequation (6) holds.

\section{A numerical example}

In this section, we present a numerical example to illustrate the theory results.

\section{Example 1 Let}

$$
A=\left(\begin{array}{ccccc}
16 & 1 & 5 & 2 & 2 \\
3 & 15 & 2 & 4 & 3 \\
2 & 2 & 18 & 1 & 4 \\
5 & 3 & 5 & 8 & 2 \\
5 & 2 & 2 & 3 & 9
\end{array}\right), \quad \beta=\{1,3\} .
$$

By calculation with Matlab 7.1, we have that

$$
\begin{aligned}
& R_{1}(A)=10 ; \quad R_{2}(A)=12 ; \quad R_{3}(A)=9 ; \quad R_{4}(A)=15 ; \quad R_{5}(A)=12 ; \\
& C_{1}(A)=15 ; \quad C_{2}(A)=8 ; \quad C_{3}(A)=14 ; \quad C_{4}(A)=10 ; \quad C_{5}(A)=11 ; \\
& \delta_{2}=2.7273 ; \quad \delta_{4}=5.4545 ; \quad \delta_{5}=3.8182 ; \\
& \delta_{2}^{T}=0.2143 ; \quad \delta_{4}^{T}=0.2143 ; \quad \delta_{5}^{T}=0.4286 .
\end{aligned}
$$

Since $\beta \in N_{r}(A)$, by Theorem 4 , any eigenvalue $\lambda$ of $A / \beta$ satisfies

$$
\lambda \in\{\lambda:|\lambda-15| \leq 9.2727\} \cup\{\lambda:|\lambda-8| \leq 9.5455\} \cup\{\lambda:|\lambda-9| \leq 8.1818\}=\Gamma_{1} .
$$

From Theorem 3 of [2], any eigenvalue $\lambda$ of $A / \beta$ satisfies

$$
\lambda \in\{\lambda:|\lambda-15| \leq 10.1250\} \cup\{\lambda:|\lambda-8| \leq 11.2500\} \cup\{\lambda:|\lambda-9| \leq 9.3750\}=\Gamma_{1}^{\prime} .
$$

Evidently, $\Gamma_{1} \subset \Gamma_{1}^{\prime}$, we use Figure 1 to show the fact. 


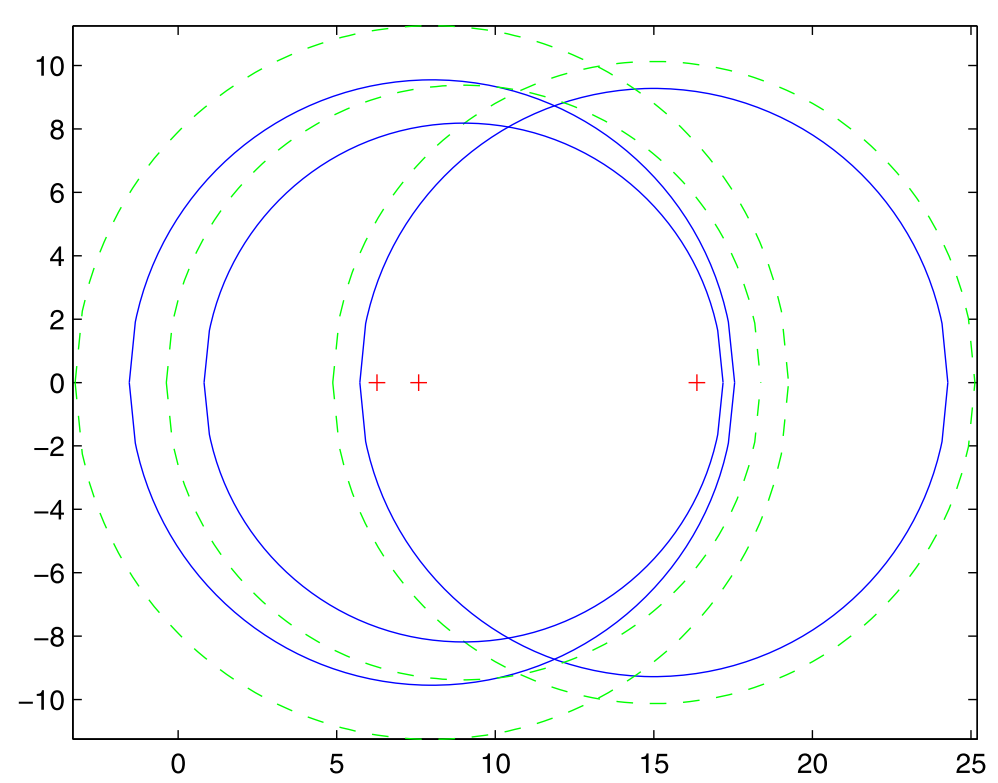

Figure 1 The blue solid line and the green dashed line denote the corresponding discs $\Gamma_{1}$ and $\Gamma_{1}^{\prime}$, respectively.

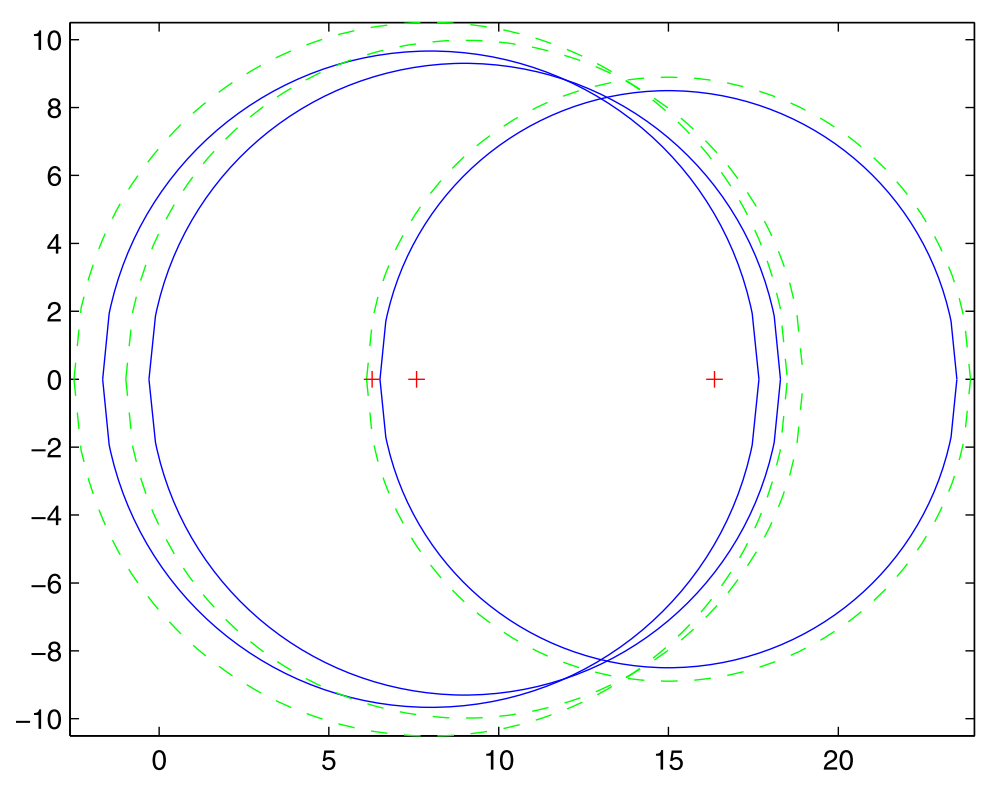

Figure 2 The blue solid line and the green dashed line denote the corresponding discs $\Gamma_{2}$ and $\Gamma_{2}^{\prime}$, respectively. 
Meanwhile, since $\beta \in N_{r}(A) \cap N_{c}(A)$, by taking $\alpha=0.5$ in Theorem 5 , any eigenvalue $\lambda$ of $A / \beta$ satisfies

$$
\lambda \in\{\lambda:|\lambda-15| \leq 8.4968\} \cup\{\lambda:|\lambda-8| \leq 9.6648\} \cup\{\lambda:|\lambda-9| \leq 9.3002\}=\Gamma_{2} .
$$

From Theorem 4 of [2], any eigenvalue $\lambda$ of $A / \beta$ satisfies

$$
\lambda \in\{\lambda:|\lambda-15| \leq 8.8939\} \cup\{\lambda:|\lambda-8| \leq 10.5067\} \cup\{\lambda:|\lambda-9| \leq 9.9804\}=\Gamma_{2}^{\prime} .
$$

Evidently, $\Gamma_{2} \subset \Gamma_{2}^{\prime}$, we use Figure 2 to show the fact.

\section{Competing interests}

The authors declare that they have no competing interests.

\section{Authors' contributions}

All authors read and approved the final manuscript.

\section{Acknowledgements}

The authors are very indebted to the referees for their valuable comments and corrections, which improved the original manuscript of this paper. This work was supported by the National Natural Science Foundation of China (71161020, 11361074) and IRTSTYN.

Received: 28 May 2013 Accepted: 26 August 2013 Published: 11 September 2013

\section{References}

1. Cvetković, LJ: A new subclass of H-matrices. Appl. Math. Comput. 208, 206-210 (2009)

2. Liu, JZ, Huang, ZJ: The Schur complements of $\gamma$-diagonally and product $\gamma$-diagonally dominant matrix and their disc separation. Linear Algebra Appl. 432, 1090-1104 (2010)

3. Liu, JZ, Li, JC, Huang, ZH, Kong, X: Some properties on Schur complement and diagonal Schur complement of some diagonally dominant matrices. Linear Algebra Appl. 428, 1009-1030 (2008)

4. Carlson, D, Markham, T: Schur complements on diagonally dominant matrices. Czechoslov. Math. J. 29, 246-251 (1979)

5. Horn, RA, Johnson, CR: Topics in Matrix Analysis. Cambridge University Press, New York (1991)

6. Ikramov, KD: Invariance of the Brauer diagonal dominance in Gaussian elimination. Vestn. Mosk. Univ., Ser. 15 Vyčisl. Mat. Kibern. 2, 91-94 (1989)

7. Smith, R: Some interlacing properties of the Schur complement of a Hermitian matrix. Linear Algebra Appl. 177, 137-144 (1992)

8. Zhang, FZ: The Schur Complement and Its Applications. Springer, New York (2005)

9. Li, B, Tsatsomeros, M: Doubly diagonally dominant matrices. Linear Algebra Appl. 261, 221-235 (1997)

10. Liu, JZ, Huang, YQ: The Schur complements of generalized doubly diagonally dominant matrices. Linear Algebra Appl. 378, 231-244 (2004)

11. Liu, JZ, Huang, YQ: Some properties on Schur complements of $H$-matrices and diagonally dominant matrices. Linear Algebra Appl. 389, 365-380 (2004)

12. Liu, JZ, Huang, ZJ: The dominant degree and disc theorem for the Schur complement. Appl. Math. Comput. 215, 4055-4066 (2010)

13. Liu, JZ, Zhang, FZ: Disc separation of the Schur complements of diagonally dominant matrices and determinantal bounds. SIAM J. Matrix Anal. Appl. 27, 665-674 (2005)

14. Liu, JZ: Some inequalities for singular values and eigenvalues of generalized Schur complements of products of matrices. Linear Algebra Appl. 286, 209-221 (1999) 